\title{
Genetic Architecture of Fusarium Head Blight Disease Resistance and Associated Traits in Nordic Spring Wheat
}

vinay kumar reddy nannuru

Norwegian University of Life Sciences Faculty of Veterinary and Biosciences: Norges miljo- og biovitenskapelige universitet Veterinaerhogskolen https://orcid.org/0000-0002-5776-8235

Susanne S. Windju

Graminor AS

\section{Tatiana Belova}

UiO Det medisinske fakultet: Universitetet i Oslo Det medisinske fakultet

Jon Arne Dieseth

Graminor AS

Muath Alsheikh

Graminor AS

\section{Yanhong Dong}

University of Minnesota

Curt A. McCartney

Morden Research and Development Centre

Maria Antonia Henriques

Morden Research and Development Centre

Hermann Buerstmayr

BOKU: Universitat fur Bodenkultur Wien

\section{Sebastian Michel}

BOKU: Universitat fur Bodenkultur Wien

\section{Theodorus H.E. Meuwissen}

Norwegian University of Life Sciences Faculty of Veterinary and Biosciences: Norges miljo- og biovitenskapelige universitet Veterinaerhogskolen

Morten Lillemo ( $\nabla$ morten.lillemo@nmbu.no)

Norwegian University of Life Sciences Faculty of Veterinary and Biosciences: Norges miljo- og biovitenskapelige universitet Veterinaerhogskolen https://orcid.org/0000-0002-8594-8794

\section{Research Article}


Keywords: Wheat, fusarium head blight, disease resistance, genome-wide association study, quantitative trait loci

Posted Date: January 26th, 2022

DOI: https://doi.org/10.21203/rs.3.rs-1273510/v1

License: (c) (i) This work is licensed under a Creative Commons Attribution 4.0 International License. Read Full License 


\section{Genetic Architecture of Fusarium Head Blight Disease Resistance 2 and Associated Traits in Nordic Spring Wheat}

3

Vinay Kumar Reddy Nannuru ${ }^{1}$, Susanne S. Windju², Tatiana Belova ${ }^{3}$, Jon Arne Dieseth ${ }^{2}$, Muath Alsheikh ${ }^{2}$, Yanhong Dong ${ }^{4}$, Curt A. McCartney ${ }^{5}$, Maria Antonia Henriques ${ }^{5}$, Hermann Buerstmayr $^{6}$, Sebastian Michel ${ }^{6}$, Theodorus H.E. Meuwissen ${ }^{7}$, Morten Lillemo ${ }^{1^{*}}$

${ }^{1}$ Department of Plant Sciences, Norwegian University of Life Sciences, Ås, 1432 Norway

${ }^{2}$ Graminor AS, Ridabu, 2322 Norway

${ }^{3}$ Centre for Molecular Medicine Norway, Faculty of Medicine, University of Oslo, Blindern, 0318 Norway

${ }^{4}$ Department of Plant Pathology, University of Minnesota, St. Paul, Minnesota, 55108 USA

${ }^{5}$ Morden Research and Development Centre, Agriculture and Agri-Food Canada, Morden, Manitoba, Canada

${ }^{6}$ Institute of Biotechnology in Plant Production, Department of Agrobiotechnology, University of Natural Resources and Life Sciences Vienna, Tulln, 3430 Austria

${ }^{7}$ Department of Animal and Aquacultural Sciences, Norwegian University of Life Sciences, Ås, 1432 Norway

*Corresponding author: morten.lillemo@ nmbu.no; Orcid-ID - 0000-0002-8594-8794

\section{Abstract}

Key message - This study identified a significant number of QTL that are associated with FHB disease resistance in NMBU spring wheat panel by conducting genome-wide association study.

Fusarium head blight (FHB) is a widely known devastating disease of wheat caused by Fusarium graminearum and other Fusarium species. FHB resistance is quantitative, highly complex and divided into several resistance types. Quantitative trait loci (QTL) that are effective against several of the resistance types give valuable contributions to resistance breeding. A spring wheat panel of 300 cultivars and breeding lines of Nordic and exotic origins was tested in artificially inoculated field trials and subjected to visual FHB assessment in the years 2013 to 2015, 2019 and 2020. Deoxynivalenol (DON) content was measured on harvested grain samples and anther extrusion (AE) was assessed in separate trials. Principal component analysis based on $35 \mathrm{~K}$ and $25 \mathrm{~K}$ SNP arrays revealed the existence of two subgroups, dividing the panel into European and exotic lines. We employed a genome-wide association study to detect QTL associated with FHB traits and identify marker-trait associations (MTAs) that consistently influenced FHB resistance. Several MTAs were found to be significant for the FHB resistance. Of importance, haplotype analysis revealed a highly significant and consisted QTL on 7A, Qfhb.nmbu.7A.2, which was further validated on an independent set of breeding lines. Breeder-friendly KASP markers were developed for this QTL that can be used in markerassisted selection. The lines in the wheat panel harboured from zero to five resistance alleles, and allele stacking showed that resistance can be significantly increased by combining several 
of these resistance alleles. This information enhances breeders' possibilities for genomic prediction and to breed cultivars with improved FHB resistance.

\section{Keywords:}

Wheat, fusarium head blight, disease resistance, genome-wide association study, quantitative trait loci

\section{Statements and Declarations:}

\section{Funding}

This work was mainly funded by the SusCrop - ERA - NET project WheatSustain with funding for this study from the Research Council of Norway (NFR grant 299615), "Bundesministerium für Nachhaltigkeit und Tourismus Österreich" (Forschungsprojekt Nr. 101402), the Genome Canada project CTAG2, and the Canadian Agricultural Partnership administered by the Canadian Wheat Research Coalition. Additional funds for the present study were received from NFR grant 199387 and Graminor.

\section{Conflicts of interest}

The authors declare no conflict of interest.

\section{Availability of data and other material}

All the data from this study i.e., both phenotypic and genotypic datasets are included with manuscript submission and other relevant information.

\section{Code Availability}

The scripts and software used for data analyses in this study are available upon request.

\section{Author contributions}

\section{VKRN SW TB JAD MA YD CM MAH HB SM TM ML.}

Funding acquisition, project administration and management, MA, ML;

Data curation, VKRN, TB, ML;

Data analysis and visualization, VKRN;

Field testing at Morden in Canada, MAH, CM;

Field testing in Norway, SW, ML, JAD;

Field testing at Tulln in Austria, SM, HB;

DON analysis, YD;

Draft writing, SW, VKRN;

Draft writing-review and editing, VKRN, SW, TB, SM, TM, ML;

Supervision, TM, ML. 


$\begin{array}{rll}83 & \text { Abbreviations: } \\ 84 & & \\ 85 & \text { AE } & \text { Anther Extrusion } \\ 86 & & \text { Days to Heading } \\ 87 & \text { DH } & \text { Days to Anthesis } \\ 88 & & \\ 89 & \text { DA } & \text { Deoxynivalenol } \\ 90 & & \text { DON corrected for Plant Height and Days to Heading } \\ 91 & \text { DON } & \text { Fusarium Head Blight } \\ 92 & & \text { FHB disease severity corrected for Plant Height and Days to Heading } \\ 93 & \text { DONcPHDH } & \\ 94 & & \text { Mega base pairs } \\ 95 & \text { FHB } & \\ 96 & & \text { Marker-Trait Associations } \\ 97 & \text { FHBcPHDH } & \\ 98 & & \text { Principal Component Analysis } \\ 99 & \text { Mbp. } & \\ 100 & & \text { Plant Height } \\ 101 & \text { MTA } & \text { Transformed DON content } \\ 102 & & \\ 103 & \text { PCA } & \end{array}$




\section{Introduction}

Wheat (Triticum aestivum L.) is one of the most widely cultivated crops with around 214 million hectares and a global production of 734 million tonnes in 2018 (FAOSTAT 2018). It is also the most cultivated cereal crop in Europe (FAOSTAT 2018). To meet the increasing demand for food and feed, breeders are continuously developing novel, more efficient germplasm with improved yield, quality, and disease resistance. To lower the risk of crop losses from plant diseases and to reduce the dependency on pesticides and their environmental impact, new cultivars are needed that combine excellent disease resistance with productivity and enduse quality. Fusarium head blight (FHB) is a devastating fungal disease affecting the wheat production worldwide. FHB can cause severe yield losses due to failed kernel development or because infected kernels are shrivelled, discoloured and low in test weight (McMullen et al. 2012). Fusarium graminearum which produces the mycotoxin deoxynivalenol (DON) is found to be the most causal agent of FHB in wheat (McMullen et al. 1997; Goswami and Kistler 2004; Hofgaard et al. 2016). Mycotoxins, such as DON may cause severe problems and is a threat to both animals and humans, reaching from feed refusal and poor weight gain in animals to immunological problems in humans (McMullen et al. 2012). Threshold levels of DON concentration set by the European Union range between $200 \mu \mathrm{g} / \mathrm{kg}$ for processed cereal based foods and baby foods for infants and young children to $750 \mu \mathrm{g} / \mathrm{kg}$ for cereals intended for direct human consumption and cereal flour. For unprocessed cereals the threshold has been set to 1250 $\mu \mathrm{g} / \mathrm{kg}$ (European Commission 2006).

Breeding for disease resistance is the most cost-effective method to control this disease (Buerstmayr et al. 2002). To develop resistant cultivars, proper understanding of resistance mechanisms is required. Resistance to FHB has been divided into active and passive resistance mechanisms. The active mechanisms are further divided into five different types; Type I: Resistance to invasion (initial infection), Type II: Resistance to pathogen spread in infected tissue, Type III: Resistance to toxin accumulation (Miller et al. 1985), Type IV: Resistance to kernel infection, and Type V: Tolerance (Schroeder and Christensen 1963; Miller et al. 1985; David Miller and Arnison 1986; Mesterhazy 1989, 1995; Mesterházy et al. 1999; Buerstmayr and Lemmens 2015). The passive resistance mechanisms are divided into four different types: Type I: plant height, Type II: presence/absence of awns, where the presence of awns increases the disease severity and otherwise, Type III: spikelet density within the head, Type IV: escape, flowering in boot stage and the ability of florets to extrude anthers (Mesterhazy 1995). The different resistance mechanisms are quantitative (complex) in nature and highly influenced by the environment, making breeding for resistance by traditional ways difficult. In addition, due to incomplete understanding of factors that influence the disease development and difficulty in efficient application, use of fungicides for controlling FHB is limited (McMullen et al. 1997; Goswami and Kistler 2004).

New marker technologies, enabling quantitative trait loci (QTL) detection, association mapping and subsequently marker detection, have the potential to increase the efficacy of resistance the complex resistance mechanisms. 
Many QTL mapping studies for FHB resistance in wheat have been performed over the past two decades, and with the single nucleotide polymorphism (SNP) marker technology, more markers are available, and it is possible to map resistance QTL more precisely in linkage maps and physical maps. These studies have been performed using different bi-parental populations and have revealed chromosomal regions harbouring FHB resistance loci. More than a decade ago, Buerstmayr et al. (2009) and Liu et al (2009) compared and assembled the information from several of these studies in maps displaying interesting chromosomal regions harbouring FHB resistance QTL, which can be further tested and potentially utilised in resistance breeding. The possibility to map the resistance QTL also led scientists more precisely to get a closer understanding of genes and mechanisms underlying the resistance trait. The most researched QTL for FHB resistance in wheat is Fhbl on chromosome arm 3BS, which is derived from the Chinese wheat cultivar Sumai 3 (Waldron et al. 1999) and has been detected in many mapping studies (Buerstmayr et al. 2009; Liu et al. 2009). It confers mainly Type II resistance (Bai et al. 2018) and can reduce disease severities by 20-50\% (Bernardo et al. 2012; Jin et al. 2013). The gene was recently cloned in two independent studies and shown to encode a histidine-rich calcium-binding protein ( $\mathrm{Li}$ et al. 2019; Su et al. 2019).

In recent years, genome-wide association studies (GWAS) for detection of FHB resistant QTL have been conducted. The benefit of these studies is the ability to capture historic recombination events and utilize collections with a wide genetic background. This increases the possibility for breeders to detect interesting QTL for resistance directly in breeding relevant germplasm and enable a targeted incorporation of resistance QTL into their breeding programs. Some of these studies have demonstrated both plant height $(\mathrm{PH})$ and anther extrusion (AE) to be negatively correlated to FHB. Skinnes et al. (2010) detected a consistent and negative correlation between $\mathrm{AE}$ and FHB disease severity, and AE and DON content in the Arina x NK93604 mapping population. A study by $\mathrm{Lu}$ et al. (2013) performed on the mapping population Shanghai3/Catbird $x$ Naxos confirmed the negative correlation between AE and FHB, and the QTL analysis further confirmed the relationship; eight out of ten AE QTL detected in the study coincided with FHB severity. Kubo et al. (2013) demonstrated that partially extruded anthers were a good source for FHB infection, while rapid extrusion and ejection of the anthers contributed to the avoidance of infection by FHB. A meta-analysis performed by Mao et al. (2010) confirmed a negative association between PH and FHB, where coincident QTL for PH and FHB were detected on chromosomes 2D, 3A, 4B, 4D and 7A.

QTL for both FHB disease severity and DON content can serve as valuable sources for disease resistance breeding in wheat. SNP markers closely linked to the resistance QTL could be further tested and used in resistance-breeding for FHB and DON resistance.

186 The aim of this study was to identify QTL for both FHB disease severity and DON in a diverse panel of 300 Nordic spring wheat lines by GWAS, to study the consistency of these QTL across environments and their association with AE. Then, to further validate these QTL to observe the potential enhancement of genomic prediction models for FHB disease resistance. 


\section{Plant material}

The germplasm used in the study was a collection of 300 hexaploid spring wheat accessions including 186 lines from Norway, 40 lines from Sweden, 37 from CIMMYT and some additional accessions from Australia, Brazil, Canada, China, Czech Republic, Denmark, Finland, France, Germany, Netherlands, Poland. Russia, Slovakia, South Africa, Switzerland, United Kingdom and USA. This panel is hereafter referred to as the NMBU spring wheat panel (Online resource 1).

As a validation panel, an independent set of 358 new breeding lines from the commercial spring wheat breeding program of Graminor was used to validate QTL identified in the NMBU spring wheat panel. This set of lines is hereafter referred to as the validation panel (Online resource $1)$.

\section{Fusarium field design, inoculation, and scoring}

\section{Field trials in Norway}

The NMBU spring wheat panel was planted in $\alpha$-lattice designs with two replicates at two locations in Norway: Vollebekk research farm at the Norwegian University of Life Sciences, Ås $\left(59^{\circ} \mathrm{N}, 90 \mathrm{~m}\right.$ above sea level) in 2013, 2014, 2015, 2019, and Staur research farm close to Hamar $\left(60^{\circ} \mathrm{N}, 153 \mathrm{~m}\right.$ above sea level) in 2014 and 2015. To ensure a high Fusarium disease pressure in these trials, spawn inoculation of the fields was performed. Grain spawn, oat kernels infected with $F$. graminearum, was prepared, and distributed in the field based on a modified protocol from Dr. Bernd Rodemann, Julius Kuhn Institute, Braunschweig using a mixture of four isolates as described by Lu et al. (2013) and Tekle et al. (2018). Mist irrigation was applied every 10-15 minutes per hour from 19:00 to 23:00 every evening in the period starting from spawn inoculation at the booting stage to 3-4 weeks after flowering to ensure high disease pressure.

FHB disease assessments were performed at the beginning of maturity, when the stems of the plants in the individual plots just started to turn yellow, but the heads were still green. At Staur research farm five random heads at three different positions in each plot were evaluated. At Vollebekk research station ten random heads at two different positions in each plot were evaluated. The evaluation was performed visually by counting the number of Fusariuminfected spikelets and dividing this by the total number of spikelets giving a percentage of infected spikelets in each plot. The field plots were harvested with a plot combine, and the level of DON in each sample was evaluated by GC-MS at the University of Minnesota mycotoxin diagnostic lab (Mirocha et al. 1998). Days to heading (DH) was scored in the same field as the Fusarium disease evaluation in every testing environment at the time when $50 \%$ of the heads in the plot had emerged. Plant height $(\mathrm{PH})$ was measured in centimeter in the fusarium nurseries at Vollebekk (in 2013, 2014, 2015, and 2019) and Staur (in 2014 and 2015). 
Anther extrusion (AE) was evaluated at Vollebekk in both the greenhouse (2013) and small field plots (2013) and in hill plots (2014, 2019 and 2020), and at Staur in hill plots (2014 and 2015) in different nurseries adjacent to the fusarium disease assessment field, avoiding the confounding effects of mist irrigation on the $\mathrm{AE}$ assessment. AE was assessed visually by a scale from 0-9, where 0 represented no anther extrusion and 9 full anther extrusion as described by Skinnes et al. (2010).

\section{Field trial in Canada}

A field trial was also conducted in Canada at Morden, Manitoba in the year 2019. The lines were planted in a single $1 \mathrm{~m}$ long row using a six-row cassette Row XL, Wintersteiger planter. $F$. graminearum corn kernel inoculum was used. The inoculum was prepared using four $F$. graminearum isolates (HSW-15-39 (3-ADON), HSW-15-87 (3-ADON), HSW-15-27 (15ADON), and HSW-15-57 (15-ADON) from Dr. Henriquez's culture collection, modifying the protocol of Gilbert and Woods (2006). Each isolate was inoculated in individual pans containing sterile corn and incubated for one month. The inoculum was dispersed at a rate of 8 g per row at 4-5 leaf stage. The inoculum application was followed by irrigation three times a week (Monday, Wednesday, and Friday) using Cadman Irrigations travellers with Briggs booms. Infected rows were rated around 21 days after anthesis for incidence and severity. The incidence was calculated using a scale from $0-100 \%$ (percent of heads with infection per plot), while the severity (0-100 \% scale) is the average amount of infection on infected heads per plot. The FHB index is the product of Incidence $\times$ Severity divided by 100 . Plant height was measured in $\mathrm{cm}$ from ground to top of heads excluding the awns. After harvest (using low wind speed on the combine to retain lightweight Fusarium-damaged kernels (FDK), samples were cleaned and a minimum of $10 \mathrm{~g}$ of well-mixed seed were ground to flour. From each replicate, $1.000 \mathrm{~g}$ of flour was used for DON (ppm) analysis using ELISA tests.

\section{Field trial in Austria}

A subset of 200 lines from the NMBU spring wheat panel was tested in a field trial conducted at the experimental station of the Department of Agrobiotechnology, Tulln in $2020\left(9^{\circ} \mathrm{N}, 177\right.$ $\mathrm{m}$ above sea level). The trial was laid out as a randomized complete block design and inoculated with the DON-producing Fusarium culmorum isolate Fc91015. Spray inoculations of each individual plot were performed with a backpack sprayer when $50 \%$ of the plants within this plot reached the flowering stage. FHB assessments and plant height was measured in a similar manner as it was in the other field trials. Anther retention was recorded with a scale of 0-20, where four central florets on five normal main spikes per plot were manually opened and the number of florets with at least one anther inside or trapped between palea and lemma were counted. This was converted to anther extrusion with the following formula: Anther Extrusion $=[1-($ Anther Retention 0-20)/20]x9. Days to anthesis $(\mathrm{DA})$ was recorded in this field trial, and was used instead of DH, to correct the FHB and DON measurements for confounding effects of earliness.

\section{Field trial of the validation panel}

The validation panel of new breeding lines was evaluated in a spawn-inoculated disease nursery with two replicates at Vollebekk research station in 2020, following the same methodology as described for the NMBU spring wheat panel above. Data was obtained for the traits $\mathrm{DH}, \mathrm{PH}$, 
FHB and DON. In addition, AE was assessed in an adjacent hill plot nursery with two replicates following the same methodology as described above for the NMBU spring wheat panel.

The validation panel was also evaluated with two replicates at the experimental station of the Department of Agrobiotechnology, Tulln in 2021 in Austria. Data was obtained for the traits DA, PH, FHB and Anther Retention. However, data for DON was not obtained from this trial. Anther Retention was converted to Anther Extrusion with the formula mentioned above.

\section{Phenotypic statistical analysis}

279

The raw data from the DON level assessment was transformed by $\log _{10}(\mathrm{DON}$ level +1$)$ to approximately obtain normally distributed values that were used throughout the entire analyses. Least square means of FHB disease severity, DON, DH, PH and AE for single environments (=trials), and across different environment for each trait were calculated using the "lme4" package (Bates et al. 2014) and "ImerTEST" (Kuznetsova et al. 2017) in R (Team 2021) using the following models:

$$
P_{i k n}=\mu+g_{i}+B_{k}+R_{n}+R: B_{k n}+e_{i k n}
$$

and

$$
P_{i j k n}=\mu+g_{i}+E_{j}+g \times E_{i j}+B_{k}+R_{n}+R: B_{k n}+e_{i j k n}
$$
each trait, where $P_{i k n}$ is the phenotype (trait value) of the $i^{\text {th }}$ variety in the $n^{\text {th }}$ replicate in the $k^{\text {th }}$ block. $\mu$ is the general mean, $g_{i}$ is the fixed effect of the $i^{\text {th }}$ variety, $B_{k}$ is the random effect of $k^{\text {th }}$ block, $R_{n}$ is the random effect of $n^{\text {th }}$ replicate, $R: B_{k n}$ is the random effect of the $k^{\text {th }}$ block within $n^{\text {th }}$ replicate, and $e_{i k n}$ is the error term. And model 2 was used to calculate LSmeans across the different environments tested for each trait, where $P_{i j k n}$ is the phenotype (trait value) of the $i^{\text {th }}$ variety in the $n^{\text {th }}$ replicate in the $k^{\text {th }}$ block in $j^{\text {th }}$ environment. $\mu$ is the general mean, $g_{i}$ is the fixed effect of the $i^{\text {th }}$ variety, $E_{j}$ is the random effect of the $j^{\text {th }}$ environment, $g \times E_{i j}$ is the random effect of the $i^{\text {th }}$ variety grown under $j^{\text {th }}$ environment (interaction), $B_{k}$ is the random effect of $k^{\text {th }}$ block, $R_{n}$ is the random effect of $n^{\text {th }}$ replicate, $R: B_{k n}$ is the random effect of the $k^{\text {th }}$ block within $n^{\text {th }}$ replicate, and $e_{i j k n}$ is the error term

FHB scorings and DON content can be confounded with variation in $\mathrm{PH}$ and $\mathrm{DH}$, to adjust for these confounding effects regressions of FHB and DON on $\mathrm{PH}$ and DH was performed and the residuals used for the further analysis, while in the case of the trials at Morden and Tulln DA was used instead of DH. The residuals are FHB corrected for PH and DH/DA (FHBcPHDH), and DON corrected for PH and DH/DA (DONcPHDH). 
Pearson correlations between the traits were calculated by Pearson method (Benesty et al. 2009) in R. Principal component analysis was performed on the available phenotypic data of FHBcPHDH, DONcPHDH and AE from all the tested environments to determine the principal coordinate values (PC scores) for each of the above-mentioned traits using the R-package "Factoextra" (Kassambara and Mundt 2017).

Broad-sense heritabilities were calculated for the across environment means of FHB, DON and $\mathrm{AE}$ based on the variance component estimates (Genetic Variance and Residual Variance) obtained from the R-package "Heritability" (Kruijer et al. 2015) in the complete panel and in the European panel using the formula:

$$
H^{2}=\frac{\sigma_{g}^{2}}{\sigma_{g}^{2}+\sigma_{E}^{2}}
$$

In the above formula, $\sigma_{g}^{2}$ is genetic variance, and $\sigma_{E}^{2}$ is the error variance.

\section{Genotypic data}

Seedlings of the NMBU spring wheat panel were grown in the greenhouse and genomic DNA was extracted from fresh young leaves using the DNeasy plant DNA extraction kit (Qiagen). The lines were genotyped with the 35K Axiom ${ }^{\circledR}$ array (Allen et al. 2017), Trait Genetics Illumina 25K SNP Chip, and in addition, some KASP and SSR markers for key agronomic and disease resistance traits (Rasheed et al. 2016) were also included in our study. The SSR markers were converted to biallelic state. Both the KASP and SSR markers were initially assigned to fictive chromosome in $35 \mathrm{~K}$ and $25 \mathrm{~K}$ genotypic datasets and later the markers which showed significant association with the traits were approximately placed close to the relative chromosomal position on the consensus map, based on physical map positions obtained from the wheat reference genome IWGSC RefSeq v1.0 (Appels et al. 2018) and LD with other significant markers. "MapChart 2.32." (Voorrips 2002) was used for graphical representation QTL positions with their intervals.

Markers were filtered based on $10 \%$ missing data and minor allele frequency of $\geq 5 \%$ in the lines. Heterozygous genotypes were regarded as missing data. Positional information was assigned using the consensus 35K SNP map (Allen et al. 2017) and Trait Genetics Illumina 25K SNP Chip. After filtering and removing redundant markers, in total 14713 and 21652 markers remained for the association mapping in $35 \mathrm{~K}$ and $25 \mathrm{~K}$ genotypic datasets (Online resource 1).

\section{The validation panel was genotyped using Trait Genetics Illumina 25K SNP Chip. After} genotyping, the markers were filtered based on the criteria mentioned above. For the present study, marker data was available for only 131 of the lines in the validation panel. The most significant SNP markers of the Qfhb.nmbu.7A.2 region were converted to KASP markers, which were used for validation purpose in the study. Primer sequences were designed using the online PolyMarker tool (http://www.polymarker.info/) (Ramirez-Gonzalez et al. 2015) or 
(https://www.cerealsdb.uk.net/cerealgenomics/CerealsDB/).

\section{Linkage Disequilibrium}

Calculation of the linkage disequilibrium (LD) over the entire genome was performed using the software TASSEL 5 (Bradbury et al. 2007). The LD was calculated with every mapped marker with allele frequencies $>0.05$, over the 21 chromosomes, in a sliding window approach, with 1000 as the window size. Squared allele frequency correlation $r^{2}$ (Hill and Weir 1988) was used to calculate pair-wise LD. The p-values were calculated using Fisher's test in TASSEL, and a threshold of $p<0.001$ was used for detecting significant LD between markers. The average genome-wide LD decay was visualized by plotting all intra-chromosomal $r^{2}$ values of all chromosomes against genetic distance in $\mathrm{cM}$ between the marker pairs. To summarize the relationship between the LD decay and genetic distance, a non-linear model described by Marroni et al. (2011) was used. For further information on the method of LD calculation, refer to Ruud et al. (2019).

\section{Genome-Wide Association Mapping}

Association analysis was performed on the traits AE, FHB corrected for $\mathrm{PH}$ and $\mathrm{DH}$ (FHBcPHDH) and DON corrected for $\mathrm{PH}$ and $\mathrm{DH}(\mathrm{DONcPHDH})$ for all the tested environments, and across-environment means and PC-scores of each trait using the "GAPIT" R package (Lipka et al. 2012). In our study, we tested several methods for GWAS, such as Mixed Linear Model, Compressed MLM, Enriched Compressed MLM and FarmCPU. Each model is briefly explained below.

MLM is a widely used single-locus model, which uses kinship matrix and population structure or principal components for controlling spurious associations (Yu et al. 2006). Compressed MLM (CMLM) by Zhang et al. (2010) is another single-locus model that clusters individuals into certain groups based on relatedness and uses genetic values as random effects, which improves statistical power compared to MLM. Enriched CMLM is an improvement of CLMM that examines alternatives to calculating kinship between groups as the average of pair-wise individual kinships ( $\mathrm{Li}$ et al. 2014). This model uses algorithms and calculates kinship, therefore classifies, and chooses the best combination for the analysis. Altogether these three single-locus models improve statistical power and reduce spurious marker-trait associations.

FarmCPU is a multi-locus model, and a modified MLM method (Liu et al. 2016). This method works iteratively with a Fixed Effect Model (FEM) and Random Effect Model (REM). Testing markers each at a time and associated markers which are estimated in REM are used as covariates in FEM. REM avoids the over-fitting problem present in FEM. In this model kinship is defined with the help of associated markers estimated by REM. At each iteration $p$-values of testing markers and associated markers are combined. 
Association mapping was performed separately for the total population of 300 spring wheat lines and for the European sub-population consisting of 237 lines using both genotype data sets 392 (35K and 25K).

393

394

Since FHB resistance is a highly quantitative trait with mostly small-effect loci (Bai et al. 2018), the Bonferroni correction or False Discovery Rate (FDR) would be too strict criteria for identifying QTL (Haikka et al. 2020). However, in this study we decided to use FDR at a rate of $5 \%$ for across environment means of each trait. And the QTL were considered significant at FDR $<5 \%$. In addition, we evaluated how often these significant QTL were detected with a less stringent threshold of $-\log 10(\mathrm{p})>3.0$ in the different environments. Those markers which were consistently detected above this threshold in two or more tested environments for a particular QTL region were considered as robust associated markers and later used for haplotype analysis and allele stacking. Quantile-Quantile (QQ) plots were inspected to identify the level at which the observed p-values started to deviate from the expected values under the null hypothesis, and for the presence of spurious associations.

\section{Haplotype analysis and allele stacking}

QTL that were consistently detected in different environments were further investigated to build haplotypes of common QTL for the traits FHBcPHDH, DONcPHDH and AE. Associated markers in those consistent QTL regions were used to construct haplotypes provided they were significant, consistent across the environments and having good LD with the highest significant marker in the QTL region. These haplotypes were tested with across environment means of corrected FHB disease severity, corrected DON content and AE from the complete panel to assess which haplotype contributes to resistance. For this purpose, pair-wise comparisons by Mann-Whitney-Wilcoxon Test (Mann and Whitney 1947) were conducted in the European and complete panel. Haplotype analysis was also carried out in the validation panel of new breeding lines from Graminor to validate the effect in an independent data set for which the haplotypes were constructed by using the same QTL detected in the NMBU spring wheat panel (25K).

Additionally, a validation was done on the haplotypes constructed based on KASP markers from the QTL Qfhb.nmbu.7A.2. These haplotypes were tested with across environment means of corrected FHB disease severity, corrected DON content and AE from the complete panel to assess which haplotype contributes to resistance.

For allele stacking, markers which were highly significant in different QTL regions were chosen for FHBcPHDH, DONcPHDH and AE. The resistant allele was determined based on the predicted allele effect of the markers associated with the QTL. Then, the lines were grouped based on the number of resistance alleles they contained. Mann-Whitney-Wilcoxon Test (Mann and Whitney 1947) was used to compare the significance of differences between the groups.

R-studio (Team 2021) was used to visualize the graphs using the following packages: "ggplot2" (Wickham et al. 2016) and "ggpubr" (Kassambara and Kassambara 2020) 


\section{Results}

\section{Phenotypic data analysis}

Across environment means of FHB severity, DON, AE, PH, and DH showed continuous variation that resembled normal distributions (Figure 1). Considerable variation was observed within the complete panel and the European panel for all the traits in all the tested environments (Supplementary figure 1).

The FHB severity was positively correlated with DON with high significance $(r=0.66, p<$ $0.0001)$, and negatively correlated with PH $(r=-0.43, p<0.0001)$ and AE $(r=-0.48, p<0.0001)$. DON was positively correlated with $\mathrm{DH}(r=0.38, p<0.0001)$, and negatively correlated with $\mathrm{PH}$ $(r=-0.31, p<0.0001)$ and $\mathrm{AE}(r=-0.47, p<0.0001)$ (Table 1$)$.

Estimated heritabilities $\left(H^{2}\right)$ of across environment means of FHB, DON and AE were 0.50, 0.69 and 0.74 in the complete panel and $0.34,0.55$ and 0.72 for the European panel respectively.

\section{Linkage disequilibrium and Population structure}

The estimated $\mathrm{r}^{2}$ for half decay was 0.22 for the NMBU spring wheat panel and the estimated genome-wide half decay distance was $1 \mathrm{cM}$ (the markers within the half decay distance of 1 $\mathrm{cM}$ were considered to be located in the same QTL on the genetic map).

Principal component analysis of the marker data confirmed the presence of two groups in the NMBU spring wheat panel, with principal component 1 (PC1) clearly separating the European lines from those originating outside Europe (Figure 2). For this reason, association analysis was done separately for the complete set of lines and the European sub-population.

\section{Genome-Wide Association Mapping}

Various GWAS models were tested on the available data, from which FarmCPU was chosen for its proven efficiency over other models in several recent studies (Liu et al. 2016; Kaler et al. 2020). Also, FarmCPU based QQ-plots showed more significant p-values compared to other models (Figure 3).

Association mapping detected significant marker-trait associations on several chromosomes for each trait. However, these significant MTAs varied from one environment location by year combination) to another. The most important and robust MTAs among the different environments and among the three traits FHBcPHDH, DONcPHDH and $\mathrm{AE}$ amounted to thirteen QTL in total. These robust QTL were significant across different tested environments and traits. They are located on the following chromosomes (the number following the chromosome number denotes different QTL region on the same chromosome, physical

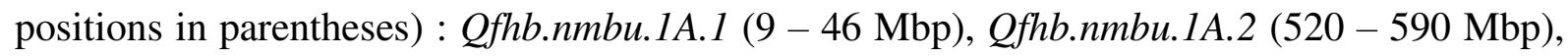
Qfhb.nmbu.3A.1 (683 - $738 \mathrm{Mbp),} \mathrm{Qfhb.nmbu.3B.1} \mathrm{(7} \mathrm{-} \mathrm{9.77} \mathrm{Mbp),} \mathrm{Qfhb.nmbu.4A.1} \mathrm{(537} \mathrm{-}$

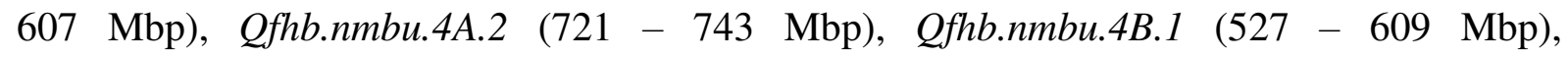
Qfhb.nmbu.5A.1 (480 - $552 \mathrm{Mbp),} \mathrm{Qfhb.nmbu.6A.I} \mathrm{(608} \mathrm{-} \mathrm{609} \mathrm{Mbp),} \mathrm{Qfhb.nmbu.6B.1} \mathrm{(630} \mathrm{-}$ 
$688 \mathrm{Mbp}$ ), Qfhb.nmbu.7A.1 (120 - $129 \mathrm{Mbp)} \mathrm{and} \mathrm{Qfhb.nmbu.7A.2} \mathrm{(670} \mathrm{-} 710 \mathrm{Mbp)} \mathrm{(Table} \mathrm{2).}$ All those detected MTAs on specified chromosomes were found using genotyping with $35 \mathrm{~K}$ and 25K SNP chips. And most of these were detected both in complete and European panel, except for two QTL regions which were specific only to the complete panel Qfhb.nmbu.1A.2 (520 - $590 \mathrm{Mbp)}$ and Qfhb.nmbu.4A.1 (537 - 607 Mbp), and one QTL region specific to the European panel on chromosome 4B, QTL Qfhb.nmbu.4B.1 (527 - 609 Mbp) (Table 2). In addition, a significant MTA was detected at the Fhbl locus on chromosome 3B, Qfhb.nmbu.3B.1 (7 - $9 \mathrm{Mbp})$. This was found significant in two environments, along with the across environment means and PC scores for DONcPHDH. This MTA was only detected in one environment (Tulln, Austria in 2020) for FHBcPHDH and none for AE (Table 2). However, this MTA was only specifically detected in the complete panel but not in the European panel.

Since the detected significant MTAs were in the near similar QTL regions (physical positions) for both the genotypic datasets, we will hereafter present further analysis using the $25 \mathrm{~K}$ data, as it is the latest data and will be used for further validations on a new set of germplasm in this study. The detailed overview of identified significant MTAs for each trait in all the tested environments, across-year means and PC scores, and in both the cases of $25 \mathrm{~K}$ and $35 \mathrm{~K}$ genotypic data are reported in Online Resource 3; Supplementary Tables 6 to 17.

Graphical representation of QTL locations and Manhattan plots from association mapping are shown in Online Resource 2 (Supplementary figure 3, 4, 5, 6, and 7).

\section{Haplotype Analysis}

Seven QTL regions were selected for haplotype analysis and the associated markers in those selected regions were used to construct haplotypes based on the criteria mentioned in the methods section. Marker information of haplotypes and number of haplotypes are shown in Online Resource 3; Supplementary Table 12 and the number of haplotype alleles varied among the selected QTL regions ranging from three to six.

For corrected FHB disease severity, only the haplotypes from the QTL region on chromosomes Qfhb.nmbu.5A.1 (480 - $552 \mathrm{Mbp)} \mathrm{and} \mathrm{Qfhb.nmbu.7A.2} \mathrm{(670} \mathrm{-} 710 \mathrm{Mbp)} \mathrm{showed} \mathrm{significant}$ effects on the disease resistance, whereas significant haplotype effects were shown for DON content in all the tested QTL regions. Haplotype effects and significant differences between the haplotypes for Qfhb.nmbu.7A.2 (670 - 710 Mbp) are shown in Figure 4. Haplotypes effects of all other tested QTL regions are shown in Online Resource 2 (Supplementary figure 8, 9, 10, 11 , and 12)

The same haplotypes from the QTL regions (Online Resource 3; Supplementary table 12) were tested on the validation panel consisting of new breeding lines tested in two different environments (Vollebekk in 2020 and Tulln in 2021). Here, only the QTL region on chromosome 7A (Qfhb.nmbu.7A.2) showed significant haplotype effects. For corrected FHB disease severity and $\mathrm{AE}$, the resistant haplotype was significantly different from other haplotypes. A clear trend in the same direction was also shown for DON content in Vollebekk 2020, although not statistically significant due to the low statistical power. Data for DON is not 
514 available from the location Tulln, 2021. Only two lines in the validation panel showed the 515 resistant haplotype, and these lines share a similar pedigree with lines having the resistant

516 haplotype on chromosomal region 7A (Qfhb.nmbu.7A.2) in the NMBU spring wheat panel 517 (Figure 5). The most significant SNP markers in this region (BS00098483_51, AX-95248570 518 and Kukri_c57593_79) were converted to breeder-friendly KASP markers and tested on the 519 NMBU spring wheat panel (Online Resource 2; Supplementary figure 14). The KASP markers 520 produced similar genotype calls as the original SNP marker data, primer sequences used for 521 these KASP markers are available in Online Resource 3 (Supplementary table 14). A few 522 anomalies were noted, which could be caused using new DNA samples for the KASP 523 genotyping. Nevertheless, the same significant haplotype effects were seen in the KASP 524 validation of complete panel and European panel as compared to the SNP chip data (Online 525 Resource 2; Supplementary figure 13)

\section{Allele Stacking}

The effect of stacking different alleles was examined on corrected FHB disease severity, corrected DON content and AE (Figure 6) in both the complete and the European panel. In the complete panel the groups ranged from zero to five resistant alleles and the markers selected for the allele stacking are shown in Online Resource 3 (Supplementary table 13). In the

532 European panel this number was reduced due to fewer lines and reduced genetic diversity, ranging from one to five resistant alleles. Overall, the lines carrying a greater number of resistant alleles (i.e., four or more) were more resistant than the groups carrying fewer resistant alleles, and significant effects of allele stacking was observed for all three traits (Figure 6). 


\section{Discussion:}

Although similar QTL were detected by all the tested models in our study, the FarmCPU model detected fewer QTL than the other models. Since FarmCPU is known to have a better control of false positives and higher power to detect true QTL compared to the other models (Liu et al. 2016; Kaler et al. 2020) we decided to base our analysis on the FarmCPU results. Overall, we detected thirteen significant QTL regions in this study. Five of them were of major importance based on different factors such as consistency across the environments and traits and considering the LD between the significant markers in the respective QTL region. Significant markers from these five QTL regions were tested for their effect on FHB disease resistance traits with the help of haplotype and allele stacking analyses. From these analyses, we were able to identify the resistant haplotypes and the lines contributing the resistance. These results were further validated by performing haplotype analysis on an independent germplasm (i.e., Validation panel), which found a QTL region Qfhb.nmbu.7A.2 (670 - 710 Mbp) on 7A to be important for improving FHB disease resistance in future breeding efforts.

\section{Heritability}

Heritabilities for FHB and DON were low when compared to AE and were further reduced in the individual analyses of the European panel, which highlights the contribution of genetic diversity in resistance by the exotic lines. The association mapping for FHB revealed several QTL over all chromosomes, but only some of those were consistent in two or more environments. For DON, the disease assessment was performed measuring the content by GCMS, which is not subjected to the same rate of error as the visual scoring of FHB in the field. The association mapping for DON also revealed several QTL in each environment, but some of the QTL were consistent in two or more environments. These findings suggest that $\mathrm{G} \times \mathrm{E}$ interactions play a significant role in the NMBU spring wheat panel tested in our study. Therefore, the QTL that were significantly detected in two or more environments for FHB and DON should be emphasized for resistance breeding in the future.

\section{Trait correlations}

Days to heading has been reported to be negatively correlated with FHB (Emrich et al. 2008), while in our study the correlation between DH and FHB was very low and negative and not significant. Correlations between DH and FHB may have confounding effect on the association mapping results, because weather conditions (humidity and temperature) during anthesis can have a huge impact on the success of FHB infection. Hence, days to heading (or anthesis) might affect the disease scores when the germplasm differ in earliness and correlations can be positive or negative, depending on weather conditions in different years. It is therefore important to correct for this factor in individual nurseries. Since we observed a positive and significant correlation between DON and DH, we decided to correct both DON and FHB for DH in the association analyses to avoid any confounding effects of this trait.

Also, PH has been reported to be associated with FHB (Mao et al. 2010; Lu et al. 2013; Kubo et al. 2013). The correlation analyses in the present study further confirm these reports as both 
FHB and DON were negatively correlated to PH. In wheat production, taller plants may lodge when fertilized and may also make use of modern machinery more difficult, the preferred plants are therefore the shorter ones. To avoid the confounding effect of PH, FHB and DON were also corrected for $\mathrm{PH}$.

Correlation of AE with FHB and DON were found to be negative and highly significant in this study. High anther extrusion has been proposed as a valuable passive resistance trait to avoid FHB infection. Many studies (Skinnes et al. 2010); Lu et al. 2013; Kubo et al. 2013; Buerstmayr and Buerstmayr 2015; He et al. 2016a, b) have reported the correlation of AE with FHB and DON and detected lower FHB and DON infections in lines with high AE, and suggested high AE to contribute to Type I resistance. These studies clearly suggested that lines with low FHB could be achieved by selecting genotypes that displayed high anther extrusion but also pointed out that lines that shed anthers well still got infected by FHB. All these findings were further confirmed by Lu et al. (2013), which reported AE to be positively correlated with Type I resistance to FHB in a biparental mapping population and also detected several AE QTL to coincide with FHB QTL. In the association mapping of the present study, we considered only the significant QTL consistent across FHB, DON, and AE to be of foremost importance and used them for validation by haplotype analysis. These findings further validate the correlation between $\mathrm{AE}$ and FHB, and DON. Therefore, searching for genotypes with high $\mathrm{AE}$, could possibly be a valuable contribution to the resistance breeding for FHB. AE is easy to score in the field and shows higher heritability than FHB and DON as demonstrated by the calculated heritabilities in this study. Fusarium resistance breeding can be enhanced by screening lines for high AE before the more laborious and costly FHB testing (Skinnes et al. 2008).

Several studies have reported correlation between FHB and DON in wheat both in segregating material and in collections of varieties with different resistance level (Bai et al. 2001; Miedaner et al. 2003; Snijders 2004; Ji et al. 2015; Hofgaard et al. 2016). The correlation between FHB and DON in the present study was also very significant and positive with a correlation coefficient of 0.66. Reports of the correlation between FHB and DON suggests a complicated relationship, Bai et al. (2001) performed thus a study with 116 cultivars and breeding lines of wheat to get further insight into this relationship. The results from their study show that cultivars moderately resistant and moderately susceptible to FHB usually had higher DON levels than resistant cultivars, but that there also were exceptions, especially for cultivars with a moderate Type II resistance. In the present study, we assume to have assessed a combination of both Type I and Type II FHB resistance in the field, because the field scoring was performed at a late stage in the development of the plants when both the initial infection (Type I) and spread (Type II) had occurred.

\section{Comparison with reported or confirmed QTL from previous studies}

Fhb1 is a well-characterized QTL descending from the Asian cultivar Sumai-3, and has been found in numerous QTL studies (Anderson et al. 2001; Liu et al. 2006; Buerstmayr et al. 2009) and is well-known for conferring Type II resistance and to a lesser extent Type I resistance (Waldron et al. 1999; Anderson et al. 2001; Buerstmayr et al. 2002, 2003; Cuthbert et al. 2006). Diagnostic markers (wgrb619_730 and wgrb619_1450) obtained from the study by Li et al. 
(2019) for $F h b 1$ were included in the genotypic data at a putative region on chromosome 3B.1 in our study, while the physical position was determined based on reference maps (Liu et al. 2006; Pumphrey et al. 2007). These diagnostic SNP markers showed MTAs with highly significant $p$-values in two tested environments and across years for the trait DONcPHDH, whereas it was only significant in one environment for FHBcPHDH and not detected in any environment for AE. These significant marker-trait associations were only detected in the complete panel but not in the European panel suggesting that resistance source for Fhbl was found among the non-European lines. Our study demonstrates that $F h b l$ is effective in providing Type III resistance, which is in line previous studies (Lemmens et al. 2005; Jiang et al. 2007a; Kluger et al. 2015). Its low allele frequency and lower heritability of FHB compared to DON likely contributed to the lack of consistent detection of an Fhbl effect for combined Type I and Type II resistance (FHB visual assessment) in the present study. However, Fhb1 is an important QTL to be considered for practical resistance breeding.

The Qfhb.nmbu.1A.1 QTL (Table 2) was located in the same region as the major QTL previously reported by Buerstmayr et al. (2009); Liu et al. (2009); and Venske et al. (2019) at the distal end of 1AS. Other studies have located FHB resistance QTL in a similar region: An FHB severity QTL from the Chinese wheat line CJ9306 was mapped to position 27.2 Mb (Jiang et al. 2007a, b) and a GWAS identified an FHB incidence QTL from Chinese elite germplasm in the same region (Zhu et al. 2020), while another recent study (Sari et al. 2018) identified an important FHB QTL for incidence and severity in the region of 1AS in T. turgidum ssp. carthlicum cv. Blackbird that agrees well with the 1A.1 QTL found in the present study.

Our study revealed $Q f h b . n m b u .7 A .2$ (Table 2) as a promising QTL for FHB resistance breeding, which we validated on new breeding lines and developed KASP assays for. The resistance haplotype at this locus explained a significant proportion of both Type I + Type II (FHB severity) and Type III (DON resistance) FHB disease resistance. A similar QTL was reported by Ruan et al. (2020) located at $671 \mathrm{Mb}$, which showed a large effect on all the examined FHB resistance related traits. Some previous studies also identified a major QTL for Type II resistance based on point inoculation in the vicinity of the Qfhb.nmbu.7A.2 region through the physical mapping of the SSR markers gwm276 and gwm262 to positions of 642.9 and 681.4 $\mathrm{Mb}$ (Semagn et al. 2007; Buerstmayr et al. 2009). Two further studies reported FHB resistance QTL in the same chromosomal area: Wu et al. (2019) identified a QTL affecting DON accumulation in the same region of an elite Chinese germplasm and Sari et al. (2018) reported QTL for FHB severity and incidence in the same region from the durum wheat inbred line DT696.

Additionally, several recent studies identified and reported QTL which are in line with the results from our current study. Venske et al. (2019) published a QTLome meta-analysis of FHB resistance, which reported validated QTL from various studies (Liu et al. 2009; Ruan et al. 2020) that are in the same chromosomal region as the QTL detected in our study such as Qfhb.nmbu.1A.1, Qfhb.nmbu.5A.1 and Qfhb.nmbu.7A.2 (Table 2). Some other QTL from our study were tested for prediction of candidate FHB-response genes based on transcriptomic and 661 candidate senes, which are within the QTL base pair intervals of Ofhb.nmbu.1A.2 662 Qfhb.nmbu.4A.1, and Qfhb.nmbu.5A.1 of our study (Table 2). 
664 Prospects for resistance and genomic breeding of European and Nordic wheat germplasm

665 Many FHB resistance QTL are derived from Asian sources (Buerstmayr et al. 2009; Steiner et 666 al. 2017). This is one of the reasons why we performed association mapping on two different 667 sets of the wheat panel in the present study, aside from the apparent existence of two sub668 populations in the NMBU spring wheat panel. This was done to enable the detection of resistance sources within adapted material (European and Nordic lines) that might not appear when performing association analysis on the complete panel. Using this approach, it was also possible to detect QTL in the exotic material that are not yet integrated into the European and Nordic breeding material. These QTL will be interesting for breeders to evaluate and possibly utilise in resistance breeding. Being strongly influenced by the environment, QTL for FHB and DON resistance need to show a consistent effect across several environments to be interesting for breeding and valuable for developing new more resistant varieties. Studying the association results for each environment many QTL turned up as significant, but when comparing every environment and their significant QTL, many of these QTL were only significant in one or two environments. Hence, we focused on consistent QTL that were significantly detected in two or more environments and choose two different significance thresholds: one for the across environment LSmeans and one for individual environment data. QTL were regarded as significant in across-environment data based on the FDR and examined for their consistency over individual environments with a more lenient significance threshold. By this criterion it was possible to assess the consistency of QTL effects across environments. It was also observed that only one QTL region was specifically significant in the European panel, while two other QTL regions were specific to the complete panel (Table 2). The QTL that are specific to complete panel should be of importance for improving the resistance in the European and Nordic material because they lack the resistant alleles from these significant QTL regions.

The QTL region (Qfhb.nmbu.7A.2) on chromosome 7A has an importance in the contribution of FHB disease resistance, which was evident from haplotype analysis of associated markers in this region both in NMBU spring wheat panel and the validation panel consisting of new breeding lines. The resistant haplotype of this QTL region was significantly more resistant from other haplotypes, showing the lowest corrected FHB disease severity and corrected DON content (Figure 4). There were five European lines with this resistant haplotype, and they are progeny of the Chinese resistant cultivar Ning 8343. Interestingly, two lines in the validation panel shared the same haplotypes and similar pedigree with the lines that showed the resistant haplotype in NMBU spring wheat panel.

Allele stacking demonstrated higher DON and FHB resistance levels among the lines that carried combinations of multiple favorable alleles from the QTL detected in our study. Most of the lines with a higher number of resistant alleles contributing to disease resistance originated from CIMMYT (Gondo-1 and lines derived from crosses with Cartbird) and China (Sumai 3, Ning 8343 and CJ9306). This list also includes some adapted lines from Norway and Sweden, including the lines whose resistance source originated from the Chinese cultivar Ning8343 (Runar//Ning8343/Brakar). This signifies the importance of the exotic resistance sources for contributing FHB resistance. Hence, making use of these resistance sources and fixing the resistant alleles in new breeding lines can improve the disease resistance considerably. 
707 We have identified thirteen QTL regions for FHB resistance traits in this GWAS, all of which 708 may have potential for a further use in resistance breeding. Most importantly the five QTL 709 regions on chromosomes $1 \mathrm{~A}, 3 \mathrm{~A}, 5 \mathrm{~A}, 6 \mathrm{~A}$ and 7A were most consistent among all the detected 710 marker-trait associations based on the criteria discussed above. The QTL Qfhb.nmbu.7A.2 was 711 additionally validated on an independent set of breeding lines, where lines carrying the resistant 712 haplotype shared a similar pedigree with the resistant lines in the NMBU spring wheat panel. 713 Moreover, breeder friendly KASP assays were developed and validated for this QTL. The 714 resistance sources and QTL identified in this study will facilitate a further genetic improvement 715 of FHB resistance in Nordic and European wheat germplasm by genomic breeding strategies, 716 which enable an accelerated stacking of multiple resistance alleles to develop new improved 717 cultivars. 


\section{Tables and Figures:}

719

720

721

722

723

724

725

726

727

728

729

730

731

732
Table 1. Pearson`s correlation coefficients between FHB severity, DON content, anther extrusion (AE), plant height $(\mathrm{PH})$ and days to heading $(\mathrm{DH})$ for across environment means (LSmeans) of the NMBU spring wheat panel.

\begin{tabular}{lllll}
\hline & AE mean & DH mean & FHB mean & PH mean \\
\hline DH mean & 0.054 & - & & \\
FHB mean & $-0.48^{* * *}$ & -0.013 & - & \\
PH mean & $0.16^{* *}$ & 0.0013 & $-0.43^{* * *}$ & - \\
DON mean & $-0.47^{* * *}$ & $0.38^{* * *}$ & $0.66^{* * *}$ & $-0.31^{* * *}$ \\
\hline
\end{tabular}

$* * * \mathrm{P}<0.0001, * * \mathrm{P}<0.001, * \mathrm{P}<0.05$ 
Table 2. Overview of the most significant and consistent QTL detected in the present GWAS study, and comparison with previously published FHB resistance QTL based on positions on the wheat reference genome assembly (RefSeq v1.0; International Wheat Genome Sequencing Consortium (IWGSC) et al. 2018)

\begin{tabular}{|c|c|c|c|c|}
\hline QTL name & $\begin{array}{l}\text { Physical map position } \\
\text { (Start - End, Mbp) }\end{array}$ & $-\log (p)$ value & NMBU Spring Wheat Panel & Published References \\
\hline Qfhb.nmbu.1A.1 & $9-46$ & $3.05-13.27$ & Complete and European panels & $\begin{array}{l}\text { Jiang et al, 2007a and 2007b; } \\
\text { Buerstmayr et al, 2009; Liu et al, 2009; } \\
\text { Sari et al, 2018; Venske et al, 2019; } \\
\text { Zhu et al, 2020 }\end{array}$ \\
\hline Qfhb.nmbu.1A.2 & $520-590$ & $3.04-8.16$ & Complete panel & Venske et al, 2019; Zheng et al, 2020 \\
\hline Qfhb.nmbu.3A.1 & $683-738$ & $3.09-12.27$ & Complete and European panels & Venske et al, 2019 \\
\hline Qfhb.nmbu.3B.1 & $7-9$ & $3.49-12.86$ & Complete panel & $\begin{array}{l}\text { Anderson et al, 2001; Liu et al, 2006; } \\
\text { Buerstmayr et al. } 2009\end{array}$ \\
\hline Qfhb.nmbu.4A.1 & $537-607$ & $3.20-9.21$ & Complete panel & Zheng et al, 2020 \\
\hline Qfhb.nmbu.4A.2 & $721-743$ & $3.09-8.40$ & Complete and European panels & In this present study \\
\hline Qfhb.nmbu.4B.1 & $527-609$ & $3.02-6.41$ & European panel & In this present study \\
\hline Qfhb.nmbu.5A.1 & $480-552$ & $3.0-12.49$ & Complete and European panels & Venske et al, 2019; Zheng et al, 2020 \\
\hline Qfhb.nmbu.6A.1 & $450-531$ & $3.06-6.66$ & Complete and European panels & In this study \\
\hline Qfhb.nmbu.6A.2 & $608-609$ & $3.09-5.73$ & Complete and European panels & Ruan et al, 2020 \\
\hline Qfhb.nmbu.6B.1 & $630-688$ & $3.03-6.55$ & Complete and European panels & In this present study \\
\hline Qfhb.nmbu.7A.1 & $120-129$ & $3.05-3.86$ & Complete and European panels & In this present study \\
\hline Qfhb.nmbu.7A.2 & $670-710$ & $3.50-25.45$ & Complete and European panels & $\begin{array}{l}\text { Semagn et al, 2007; Buerstmayr et al, 2009; } \\
\text { Sari et al, 2018; Wu et al, 2019; } \\
\text { Ruan et al, } 2020\end{array}$ \\
\hline
\end{tabular}


Figure 1. Histogram distributions based on the mean frequency data over all testing environments for AE, DH, FHB, PH and tDON for the NMBU spring wheat panel.

Figure 2. Principal Component Analysis based on the $25 \mathrm{~K}$ data showing the population structure of NMBU spring-wheat panel, which is divided mainly into two groups - European and Others (lines from outside the Europe such as from CIMMYT, China, USA etc.).

Figure 3. QQ-plots of FHBcPHDHmean, DONcPHDHmean and AEmean for different GWAS models - GLM, CMLM, ECMLM and FarmCPU.

Figure 4: Boxplot showing the haplotype effect of QTL on chromosome 7A based on the a) mean AE, b) mean corrected DON content, c) mean corrected FHB disease severity of complete panel, and d) mean AE, e) mean corrected DON content, f) mean corrected FHB disease severity of European panel. Wilcoxon method is used for pair-wise comparisons $(* * * \mathrm{P}<$ $\left.0.0001,{ }^{*} * \mathrm{P}<0.001, * \mathrm{P}<0.05, \mathrm{~ns}>0.05\right)$. Non-significant comparisons are not shown in this figure.

Figure 5: Boxplot showing the haplotype effect of QTL on chromosome 7A based on the a) $\mathrm{AE}, \mathrm{b})$ corrected DON content, c) corrected FHB disease severity of validation panel at Vollebekk in 2020 and d) AE, e) corrected FHB disease severity of validation panel at Tulln in 2021. Wilcoxon method is used for pair-wise comparisons ( $* * * \mathrm{P}<0.0001, * * \mathrm{P}<0.001, * \mathrm{P}<$ $0.05, \mathrm{~ns}>0.05)$. Non-significant comparisons are not shown in this figure.

Figure 6: Boxplot showing the effect of number of stacked resistant alleles based on the a) mean $\mathrm{AE}, \mathrm{b}$ ) mean corrected DON content, c) mean corrected FHB disease severity of complete panel, and d) mean AE, e) mean corrected DON content, f) mean corrected FHB disease severity of European panel. Wilcoxon method is used for pair-wise comparisons $(* * * \mathrm{P}<0.0001$, $* * \mathrm{P}<0.001, * \mathrm{P}<0.05, \mathrm{~ns}>0.05)$. Non-significant comparisons are not shown in this figure.

769

770

771

772

773

774

775

776

777

778

779

780

781

\section{Supplementary Information:}

Online Resource 1. Phenotypic data and Genotypic data used in the study. Phenotypic data Information of NMBU spring wheat panel including genotype ID, names, country of origin. and the raw and corrected data for each genotype in various environments for each trait for NMBU spring wheat panel and Validation panel. Genotypic data $-35 \mathrm{~K}$ and $25 \mathrm{~K}$ data for 14713 and 21653 SNPs, respectively, of 296 lines for NMBU spring wheat panel including SNP ID, chromosome, physical position, - and 25K data for 19679 SNPs of 131 lines of the validation panel, including SNP ID, chromosome, physical position.

Online Resource 2. Supplementary figures including the frequency distributions of all the traits tested in different environments, correlations between the traits, Manhattan plots of GWAS with supporting QQ-plots, boxplots showing the haplotype effects of QTL validation, KASP validation and cluster plots of KASP markers. 
782 Online Resource 3. Supplementary tables containing the information of MTAs including 783 marker name, chromosome number, physical position, p-value, -log10(p-value), FDR-adjusted 784 value, starting position and ending position on chromosomes in bp, information of selected 785 significant markers used for allele stacking and haplotype analysis and information about KASP 786 marker primer sequences. 


\section{References:}

Allen AM, Winfield MO, Burridge AJ, et al (2017) Characterization of a Wheat Breeders' Array suitable for high-throughput SNP genotyping of global accessions of hexaploid bread wheat ( Triticum aestivum ). Plant Biotechnol J 15:390-401. https://doi.org/10.1111/pbi.12635

Anderson JA, Stack R, Liu S, et al (2001) DNA markers for Fusarium head blight resistance QTLs in two wheat populations. Theor Appl Genet 102:1164-1168. https://doi.org/10.1007/s001220000509

Appels R, Eversole K, Stein N, et al (2018) Shifting the limits in wheat research and breeding using a fully annotated reference genome. Science 361:6403. https://www.science.org/doi/10.1126/science.aar7191

Bai G, Su Z, Cai J (2018) Wheat resistance to Fusarium head blight. Can J Plant Pathol 40:336-346. https://doi.org/10.1080/07060661.2018.1476411

Bai G-H, Plattner R, Desjardins A, Kolb F (2001) Resistance to Fusarium head blight and deoxynivalenol accumulation in wheat. Plant Breed 120:1-6. https://doi.org/10.1046/j.1439-0523.2001.00562.x

Bates, Douglas, Martin Mächler, Ben Bolker, and Steve Walker. 2015. "Fitting Linear MixedEffects Models Using Lme4”. Journal of Statistical Software 67 (1):1-48. https://doi.org/10.18637/jss.v067.i01

Benesty J., Chen J., Huang Y., Cohen I. (2009) Pearson Correlation Coefficient. In: Noise Reduction in Speech Processing. Springer Topics in Signal Processing, vol 2. Springer, Berlin, Heidelberg. https://doi.org/10.1007/978-3-642-00296-0_5

Bernardo AN, Ma H, Zhang D, Bai G (2012) Single nucleotide polymorphism in wheat chromosome region harboring Fhb1 for Fusarium head blight resistance. Mol Breed 29:477-488. https://doi.org/10.1007/s11032-011-9565-y

Bradbury PJ, Zhang Z, Kroon DE, et al (2007) TASSEL: software for association mapping of complex traits in diverse samples. Bioinformatics 23:2633-2635. https://doi.org/10.1093/bioinformatics/btm308

Buerstmayr H, Ban T, Anderson JA (2009) QTL mapping and marker-assisted selection for Fusarium head blight resistance in wheat: a review. Plant Breed 128:1-26. https://doi.org/10.1111/j.1439-0523.2008.01550.x

Buerstmayr H, Lemmens M (2015) Breeding healthy cereals: genetic improvement of Fusarium resistance and consequences for mycotoxins. World Mycotoxin J 8:591602. https://doi.org/10.3920/WMJ2015.1889 
Buerstmayr H, Lemmens M, Hartl L, et al (2002) Molecular mapping of QTLs for Fusarium head blight resistance in spring wheat. I. Resistance to fungal spread (Type II resistance). Theor Appl Genet 104:84-91. https://doi.org/10.1007/s001220200009

Buerstmayr H, Steiner B, Hartl L, et al (2003) Molecular mapping of QTLs for Fusarium head blight resistance in spring wheat. II. Resistance to fungal penetration and spread. Theor Appl Genet 107:503-508. https://doi.org/10.1007/s00122-003-1272-6

Buerstmayr M, Buerstmayr H (2015) Comparative mapping of quantitative trait loci for Fusarium head blight resistance and anther retention in the winter wheat population Capo $\times$ Arina. Theor Appl Genet 128:1519-1530. https://doi.org/10.1007/s00122-0152527-8

Cuthbert PA, Somers DJ, Thomas J, et al (2006) Fine mapping Fhb1, a major gene controlling fusarium head blight resistance in bread wheat (Triticum aestivum L.). Theor Appl Genet 112:1465-1472. https://doi.org/10.1007/s00122-006-0249-7

David Miller J, Arnison PG (1986) Degradation of deoxynivalenol by suspension cultures of the Fusarium head blight resistant wheat cultivar Frontana. Can J Plant Pathol 8:147150. https://doi.org/10.1080/07060668609501818

Emrich K, Wilde F, Miedaner T, Piepho HP (2008) REML approach for adjusting the Fusarium head blight rating to a phenological date in inoculated selection experiments of wheat. Theor Appl Genet 117:65-73. https://doi.org/10.1007/s00122-008-0753-z

European Commission (2006) Commission Regulation (EC) No 1881/2006 of 19 December 2006 setting maximum levels for certain contaminants in foodstuffs. J Eur Union $364: 5$

FAOSTAT 2018 FAOSTAT (Food and Agriculture Organization of the United Nations) 2018

Goswami RS, Kistler HC (2004) Heading for disaster: Fusarium graminearum on cereal crops: Fusarium graminearum on cereal crops. Mol Plant Pathol 5:515-525. https://doi.org/10.1111/j.1364-3703.2004.00252.x

Haikka H, Manninen O, Hautsalo J, et al (2020) Genome-wide Association Study and Genomic Prediction for Fusarium graminearum Resistance Traits in Nordic Oat (Avena sativa L.). Agronomy 10:174. https://doi.org/10.3390/agronomy10020174

He X, Lillemo M, Shi J, et al (2016a) QTL characterization of Fusarium head blight resistance in CIMMYT bread wheat line Soru\# 1. PloS One 11:e0158052. https://doi.org/10.1371/journal.pone.0158052

He X, Singh PK, Dreisigacker S, et al (2016b) Dwarfing genes Rht-B1b and Rht-D1b are associated with both type I FHB susceptibility and low anther extrusion in two bread wheat populations. PloS One 11:e0162499. https://doi.org/10.1371/journal.pone.0162499 
Hill W, Weir B (1988) Variances and covariances of squared linkage disequilibria in finite populations. Theor Popul Biol 33:54-78. https://doi.org/10.1016/00405809(88)90004-4

Hofgaard IS, Aamot HU, Torp T, et al (2016) Associations between Fusarium species and mycotoxins in oats and spring wheat from farmers' fields in Norway over a six-year period. World Mycotoxin J 9:365-378. https://doi.org/10.3920/WMJ2015.2003

Ji F, Wu J, Zhao H, et al (2015) Relationship of deoxynivalenol content in grain, chaff, and straw with Fusarium head blight severity in wheat varieties with various levels of resistance. Toxins 7:728-742. https://doi.org/10.3390/toxins7030728

Jiang G-L, Dong Y, Shi J, Ward RW (2007a) QTL analysis of resistance to Fusarium head blight in the novel wheat germplasm CJ 9306. II. Resistance to deoxynivalenol accumulation and grain yield loss. Theor Appl Genet 115:1043-1052. https://doi.org/10.1007/s00122-007-0630-1

Jiang G-L, Shi J, Ward RW (2007b) QTL analysis of resistance to Fusarium head blight in the novel wheat germplasm CJ 9306. I. Resistance to fungal spread. Theor Appl Genet 116:3-13. https://doi.org/10.1007/s00122-007-0641-y

Jin F, Zhang D, Bockus W, et al (2013) Fusarium Head Blight Resistance in U.S. Winter Wheat Cultivars and Elite Breeding Lines. Crop Sci 53:2006-2013. https://doi.org/10.2135/cropsci2012.09.0531

Kaler AS, Gillman JD, Beissinger T, Purcell LC (2020) Comparing Different Statistical Models and Multiple Testing Corrections for Association Mapping in Soybean and Maize. Front Plant Sci 10:1794. https://doi.org/10.3389/fpls.2019.01794

Kassambara A, Kassambara MA (2020) Package 'ggpubr'

Kassambara A, Mundt F (2017) Package ‘factoextra.' Extr Vis Results Multivar Data Anal 76:

Kluger B, Bueschl C, Lemmens M, et al (2015) Biotransformation of the Mycotoxin Deoxynivalenol in Fusarium Resistant and Susceptible Near Isogenic Wheat Lines. PLOS ONE 10:e0119656. https://doi.org/10.1371/journal.pone.0119656

Kruijer W, Boer MP, Malosetti M, et al (2015) Marker-Based Estimation of Heritability in Immortal Populations. Genetics 199:379-398. https://doi.org/10.1534/genetics.114.167916

Kubo K, Fujita M, Kawada N, et al (2013) Minor Differences in Anther Extrusion Affect Resistance to Fusarium Head Blight in Wheat. J Phytopathol 161:308-314. https://doi.org/10.1111/jph.12060

Kuznetsova A, Brockhoff PB, Christensen RH (2017) lmerTest package: tests in linear mixed effects models. J Stat Softw 82:1-26

Lemmens M, Scholz U, Berthiller F, et al (2005) The Ability to Detoxify the Mycotoxin Deoxynivalenol Colocalizes With a Major Quantitative Trait Locus for Fusarium 
Head Blight Resistance in Wheat. Mol Plant-Microbe Interactions ${ }^{\circledR} 18: 1318-1324$. https://doi.org/10.1094/MPMI-18-1318

900

901

902

903

904

905

906

907

908

909

910

911

912

913

914

915

916

917

918

919

920

921

922

923

924

925

926

927

928

929

930

931

932

933

934

Li G, Zhou J, Jia H, et al (2019) Mutation of a histidine-rich calcium-binding-protein gene in wheat confers resistance to Fusarium head blight. Nat Genet 51:1106-1112. https://doi.org/10.1038/s41588-019-0426-7

Li M, Liu X, Bradbury P, et al (2014) Enrichment of statistical power for genome-wide association studies. BMC Biol 12:73. https://doi.org/10.1186/s12915-014-0073-5

Lipka AE, Tian F, Wang Q, et al (2012) GAPIT: genome association and prediction integrated tool. Bioinformatics 28:2397-2399

Liu S, Hall MD, Griffey CA, McKendry AL (2009) Meta-Analysis of QTL Associated with Fusarium Head Blight Resistance in Wheat. Crop Sci 49:1955-1968. https://doi.org/10.2135/cropsci2009.03.0115

Liu S, Zhang X, Pumphrey MO, et al (2006) Complex microcolinearity among wheat, rice, and barley revealed by fine mapping of the genomic region harboring a major QTL for resistance to Fusarium head blight in wheat. Funct Integr Genomics 6:83-89. https://doi.org/10.1007/s10142-005-0007-y

Liu X, Huang M, Fan B, et al (2016) Iterative Usage of Fixed and Random Effect Models for Powerful and Efficient Genome-Wide Association Studies. PLOS Genet 12:e1005767. https://doi.org/10.1371/journal.pgen.1005767

Lu Q, Lillemo M, Skinnes H, et al (2013) Anther extrusion and plant height are associated with Type I resistance to Fusarium head blight in bread wheat line 'Shanghai3/Catbird.' Theor Appl Genet 126:317-334. https://doi.org/10.1007/s00122-012-19819

Mann HB, Whitney DR (1947) On a test of whether one of two random variables is stochastically larger than the other. Ann Math Stat 50-60

Mao S-L, Wei Y-M, Cao W, et al (2010) Confirmation of the relationship between plant height and Fusarium head blight resistance in wheat (Triticum aestivum L.) by QTL meta-analysis. Euphytica 174:343-356. https://doi.org/10.1007/s10681-010-0128-9

Marroni F, Pinosio S, Zaina G, et al (2011) Nucleotide diversity and linkage disequilibrium in Populus nigra cinnamyl alcohol dehydrogenase (CAD4) gene. Tree Genet Genomes 7:1011-1023. https://doi.org/10.1007/s11295-011-0391-5

McMullen M, Bergstrom G, De Wolf E, et al (2012) A unified effort to fight an enemy of wheat and barley: Fusarium head blight. Plant Dis 96:1712-1728. https://doi.org/10.1094/PDIS-03-12-0291-FE

McMullen M, Jones R, Gallenberg D (1997) Scab of Wheat and Barley: A Re-emerging Disease of Devastating Impact. Plant Dis 81:1340-1348. https://doi.org/10.1094/PDIS.1997.81.12.1340 
Mesterhazy A (1989) Progress in breeding of wheat and corn genotypes not susceptible to infection by Fusaria. In: Fusarium. Elsevier, pp 357-386. https://doi.org/10.1016/B978-0-444-87468-9.50026-2

Mesterhazy A (1995) Types and components of resistance to Fusarium head blight of wheat. Plant Breed 114:377-386. https://doi.org/10.1111/j.1439-0523.1995.tb00816.x

Mesterházy Á, Bartók T, Mirocha CG, Komoróczy R (1999) Nature of wheat resistance to Fusarium head blight and the role of deoxynivalenol for breeding. Plant Breed 118:97-110. https://doi.org/10.1046/j.1439-0523.1999.118002097.x

Miedaner T, Schneider B, Geiger HH (2003) Deoxynivalenol (DON) Content and Fusarium Head Blight Resistance in Segregating Populations of Winter Rye and Winter Wheat. CROP Sci 43:8. https://doi.org/10.2135/cropsci2003.5190

Miller J, Young J, Sampson D (1985) Deoxynivalenol and Fusarium head blight resistance in spring cereals. J Phytopathol 113:359-367. https://doi.org/10.1111/j.14390434.1985.tb04837.x

Mirocha CJ, Kolaczkowski E, Xie W, et al (1998) Analysis of Deoxynivalenol and Its Derivatives (Batch and Single Kernel) Using Gas Chromatography/Mass Spectrometry. J Agric Food Chem 46:1414-1418. https://doi.org/10.1021/jf970857o

Pumphrey MO, Bernardo R, Anderson JA (2007) Validating the Fhb1 QTL for Fusarium Head Blight Resistance in Near-Isogenic Wheat Lines Developed from Breeding Populations. Crop Sci 47:200-206. https://doi.org/10.2135/cropsci2006.03.0206

Ramirez-Gonzalez RH, Uauy C, Caccamo M (2015) PolyMarker: A fast polyploid primer design pipeline: Fig. 1. Bioinformatics 31:2038-2039. https://doi.org/10.1093/bioinformatics/btv069

Rasheed A, Wen W, Gao F, et al (2016) Development and validation of KASP assays for genes underpinning key economic traits in bread wheat. Theor Appl Genet 129:18431860. https://doi.org/10.1007/s00122-016-2743-x

Ruan Y, Zhang W, Knox RE, et al (2020) Characterization of the Genetic Architecture for Fusarium Head Blight Resistance in Durum Wheat: The Complex Association of Resistance, Flowering Time, and Height Genes. Front Plant Sci 11:592064. https://doi.org/10.3389/fpls.2020.592064

Ruud AK, Dieseth JA, Ficke A, et al (2019) Genome-wide association mapping of resistance to Septoria nodorum leaf blotch in a nordic spring wheat collection. Plant Genome 12. https://doi.org/10.3835/plantgenome2018.12.0105

Sari E, Berraies S, Knox RE, et al (2018) High density genetic mapping of Fusarium head blight resistance QTL in tetraploid wheat. PLOS ONE 13:e0204362. https://doi.org/10.1371/journal.pone.0204362

Schroeder H, Christensen J (1963) Factors affecting resistance of wheat to scab caused by Gibberella zeae. Phytopathology 53:831-838 
Semagn K, Skinnes H, Bjørnstad Å, et al (2007) Quantitative Trait Loci Controlling Fusarium Head Blight Resistance and Low Deoxynivalenol Content in Hexaploid Wheat Population from 'Arina' and NK93604. Crop Sci 47:294-303. https://doi.org/10.2135/cropsci2006.02.0095

Skinnes H, Semagn K, Tarkegne Y, et al (2010) The inheritance of anther extrusion in hexaploid wheat and its relationship to Fusarium head blight resistance and deoxynivalenol content. Plant Breed 129:149-155. https://doi.org/10.1111/j.14390523.2009.01731.x

Skinnes H, Tarkegne Y, Dieseth J, Bjørnstad A (2008) Associations between anther extrusion and Fusarium Head Blight in European wheat. Cereal Res Commun 36:223-231. https://doi.org/10.1556/CRC.36.2008.Suppl.B.19

Snijders CHA (2004) Resistance in wheat to Fusarium infection and trichothecene formation. Toxicol Lett 153:37-46. https://doi.org/10.1016/j.toxlet.2004.04.044

Steiner B, Buerstmayr M, Michel S, et al (2017) Breeding strategies and advances in line selection for Fusarium head blight resistance in wheat. Trop Plant Pathol 42:165-174. https://doi.org/10.1007/s40858-017-0127-7

Su Z, Bernardo A, Tian B, et al (2019) A deletion mutation in TaHRC confers Fhb1 resistance to Fusarium head blight in wheat. Nat Genet 51:1099-1105. https://doi.org/10.1038/s41588-019-0425-8

Team RC (2021) R: A language and environment for statistical computing (R Version 4.0. 3, R Foundation for Statistical Computing, Vienna, Austria, 2020)

Tekle S, Lillemo M, Skinnes H, et al (2018) Screening of Oat Accessions for Fusarium Head Blight Resistance Using Spawn-Inoculated Field Experiments. Crop Sci 58:143-151. https://doi.org/10.2135/cropsci2017.04.0264

Venske E, dos Santos RS, Farias D da R, et al (2019) Meta-Analysis of the QTLome of Fusarium Head Blight Resistance in Bread Wheat: Refining the Current Puzzle. Front Plant Sci 10:727. https://doi.org/10.3389/fpls.2019.00727

Voorrips RE (2002) MapChart: Software for the Graphical Presentation of Linkage Maps and QTLs. J Hered 93:77-78. https://doi.org/10.1093/jhered/93.1.77

Waldron BL, Moreno-Sevilla B, Anderson JA, et al (1999) RFLP Mapping of QTL for Fusarium Head Blight Resistance in Wheat. Crop Sci 39:805-811. https://doi.org/10.2135/cropsci1999.0011183X003900030032x

Wickham H, Chang W, Wickham MH (2016) Package 'ggplot2.' Create Elegant Data Vis Using Gramm Graph Version 2:1-189

Wu L, Zhang Y, He Y, et al (2019) Genome-Wide Association Mapping of Resistance to Fusarium Head Blight Spread and Deoxynivalenol Accumulation in Chinese Elite Wheat Germplasm. Phytopathology® 109:1208-1216. https://doi.org/10.1094/PHYTO-12-18-0484-R 
1011 Yu J, Pressoir G, Briggs WH, et al (2006) A unified mixed-model method for association mapping that accounts for multiple levels of relatedness. Nat Genet 38:203-208. https://doi.org/10.1038/ng1702

1014 Zhang Z, Ersoz E, Lai C-Q, et al (2010) Mixed linear model approach adapted for genomewide association studies. Nat Genet 42:355-360. https://doi.org/10.1038/ng.546

1016 Zheng T, Hua C, Li L, et al (2020) Integration of meta-QTL discovery with omics: Towards a molecular breeding platform for improving wheat resistance to Fusarium head blight. Crop J S221451412030177X. https://doi.org/10.1016/j.cj.2020.10.006

Zhu Z, Chen L, Zhang W, et al (2020) Genome-Wide Association Analysis of Fusarium Head Blight Resistance in Chinese Elite Wheat Lines. Front Plant Sci 11:206. https://doi.org/10.3389/fpls.2020.00206 
Figures

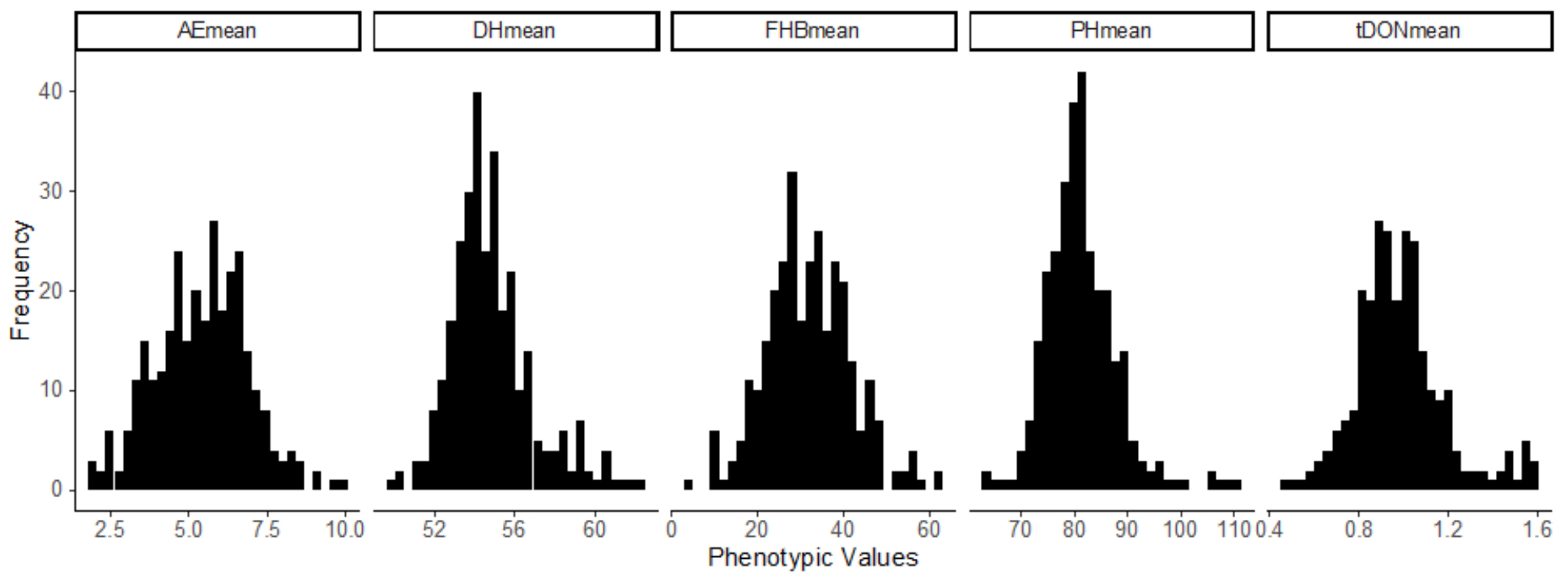

Figure 1

Histogram distributions based on the mean frequency data over all testing environments for $A E, D H, F H B$, $\mathrm{PH}$ and tDON for the NMBU spring wheat panel.

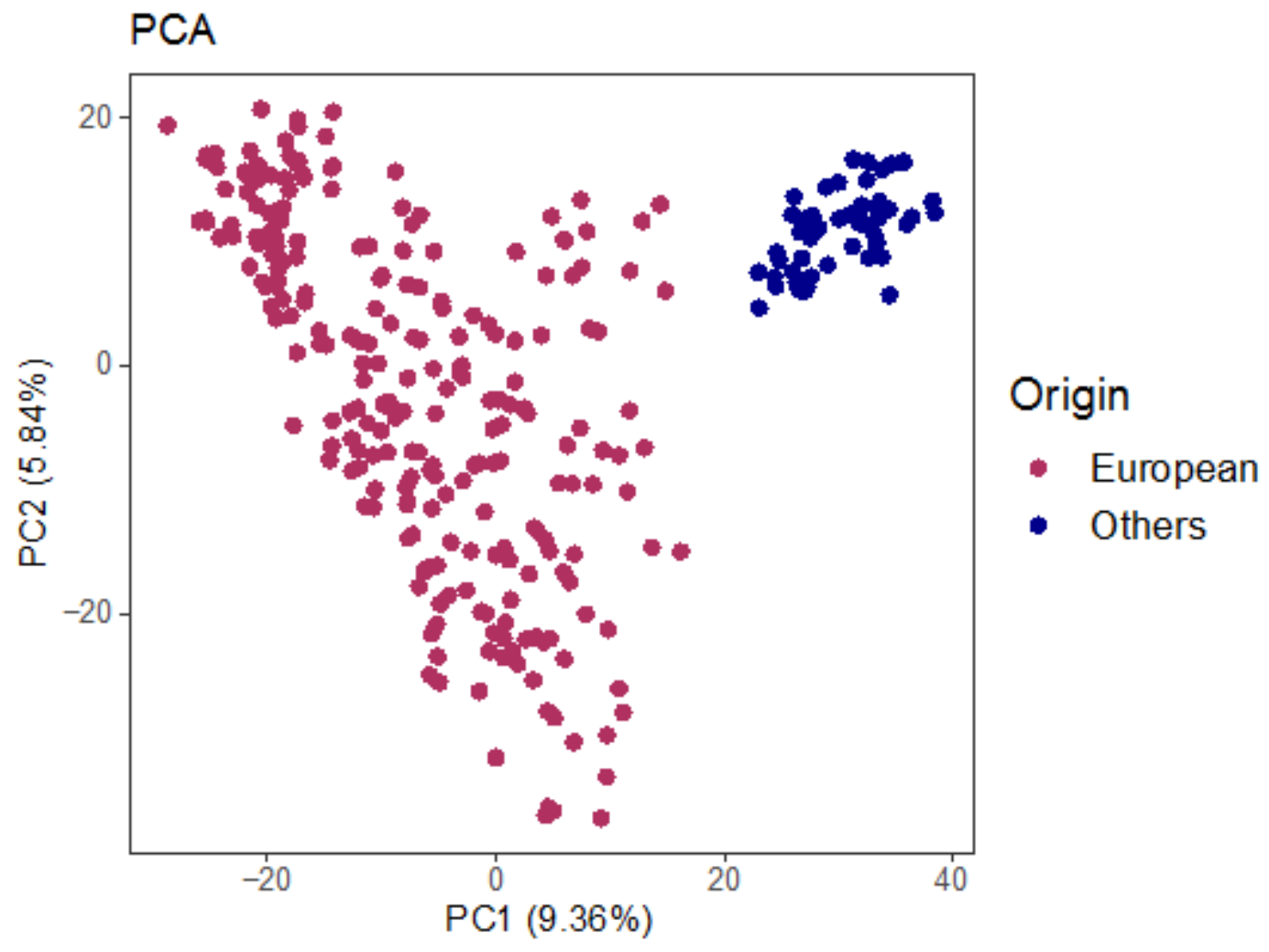

Figure 2 
Principal Component Analysis based on the $25 \mathrm{~K}$ data showing the population structure of NMBU springwheat panel, which is divided mainly into two groups - European and Others (lines from outside the Europe such as from CIMMYT, China, USA etc.).

(a)

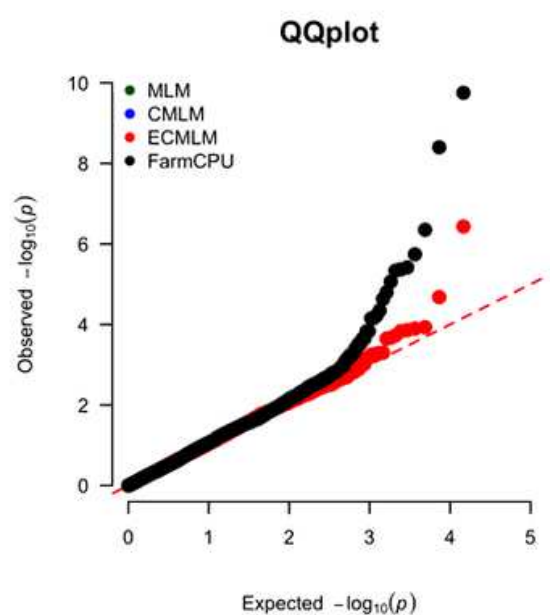

(b)

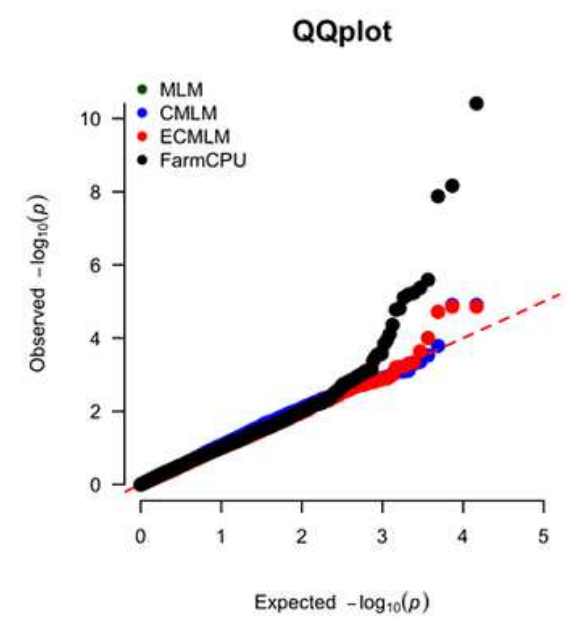

(c)

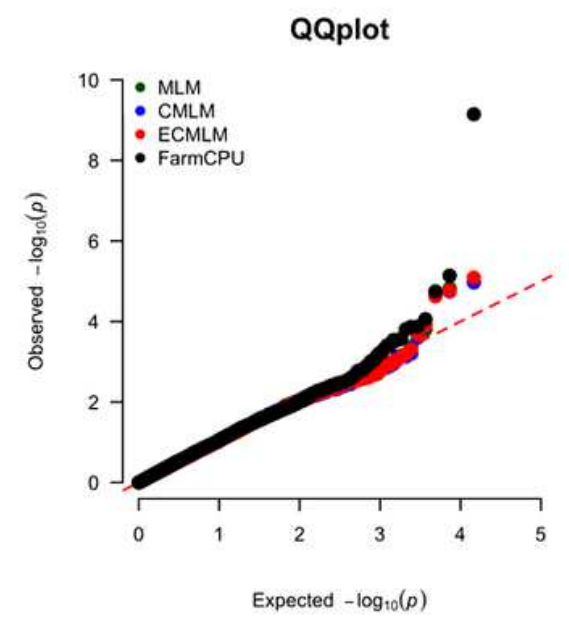

\section{Figure 3}

QQ-plots of FHBcPHDHmean, DONcPHDHmean and AEmean for different GWAS models - GLM, CMLM, ECMLM and FarmCPU.
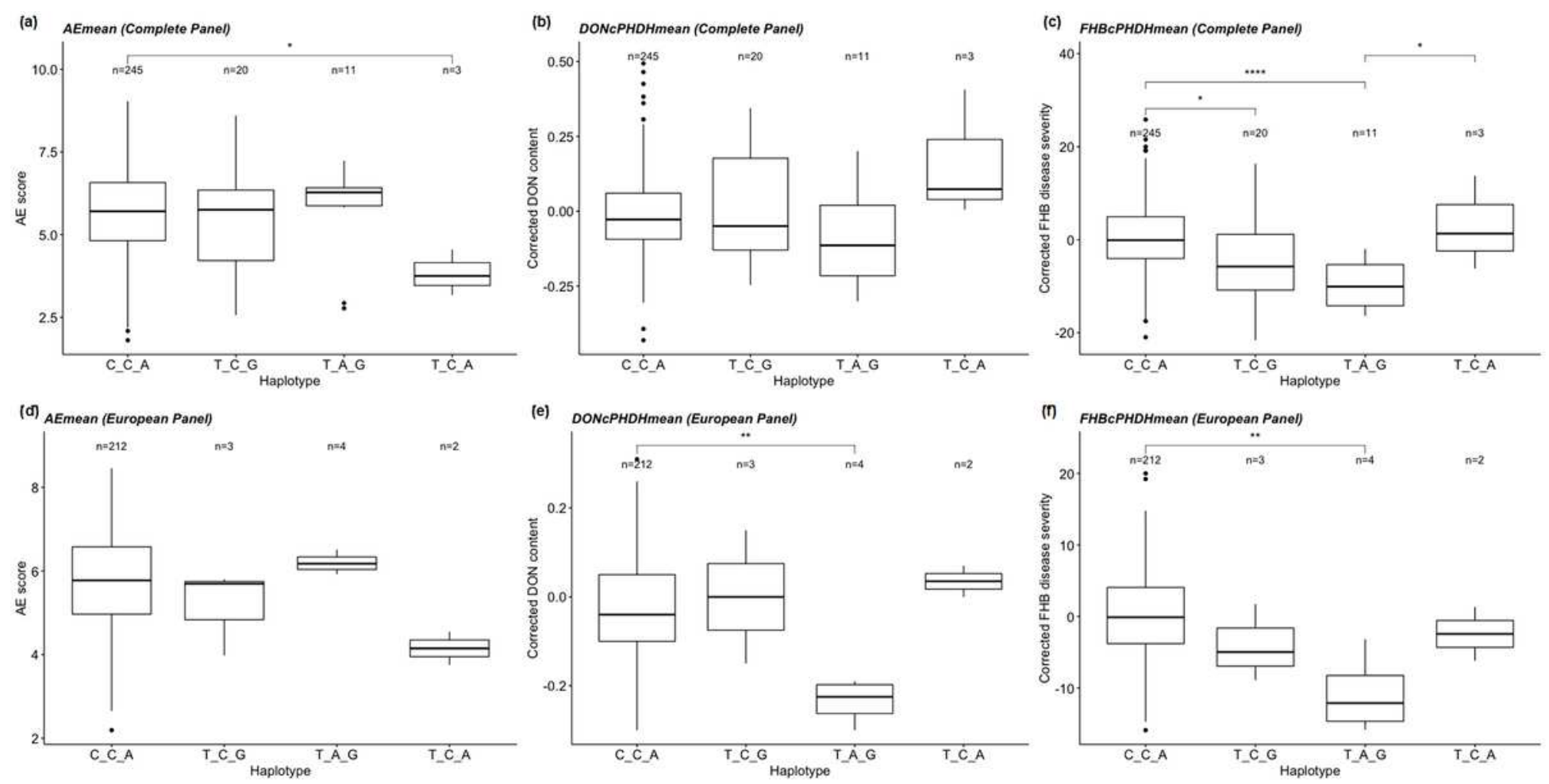


\section{Figure 4}

Boxplot showing the haplotype effect of QTL on chromosome 7A based on the a) mean AE, b) mean corrected DON content, c) mean corrected FHB disease severity of complete panel, and d) mean AE, e) mean corrected DON content, f) mean corrected FHB disease severity of European panel. Wilcoxon method is used for pair-wise comparisons ( ${ }^{\star *} \mathrm{P}<0.0001$, ${ }^{\star *} \mathrm{P}<0.001$, $* \mathrm{P}<0.05$, ns $>0.05$ ). Nonsignificant comparisons are not shown in this figure.
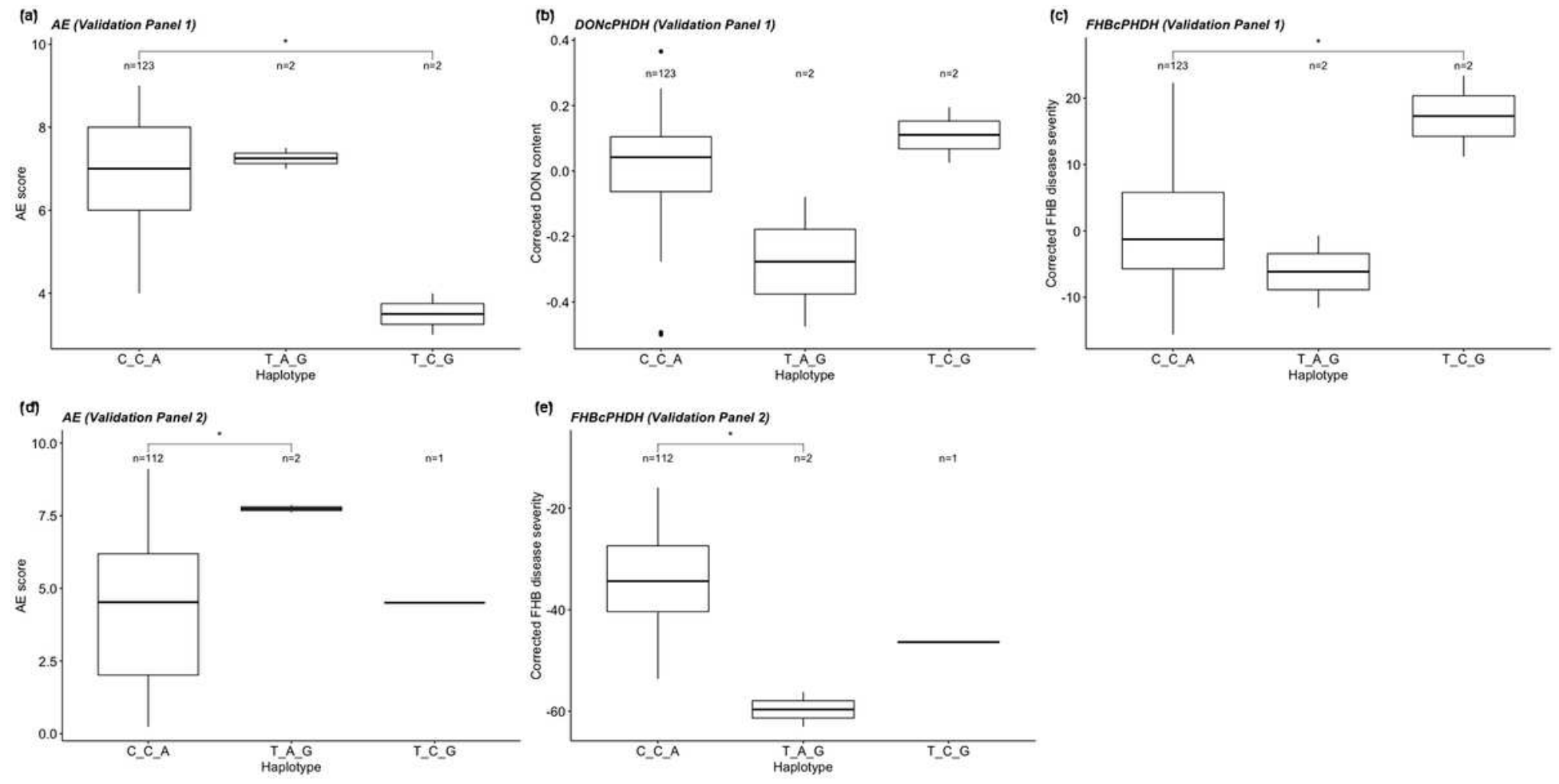

\section{Figure 5}

Boxplot showing the haplotype effect of QTL on chromosome 7A based on the a) AE, b) corrected DON content, c) corrected FHB disease severity of validation panel at Vollebekk in 2020 and d) AE, e) corrected FHB disease severity of validation panel at Tulln in 2021. Wilcoxon method is used for pair-wise comparisons ( ${ }^{\star \star *} \mathrm{P}<0.0001,{ }^{\star *} \mathrm{P}<0.001,{ }^{*} \mathrm{P}<0.05$, ns $>0.05$ ). Non-significant comparisons are not shown in this figure. 
(a)

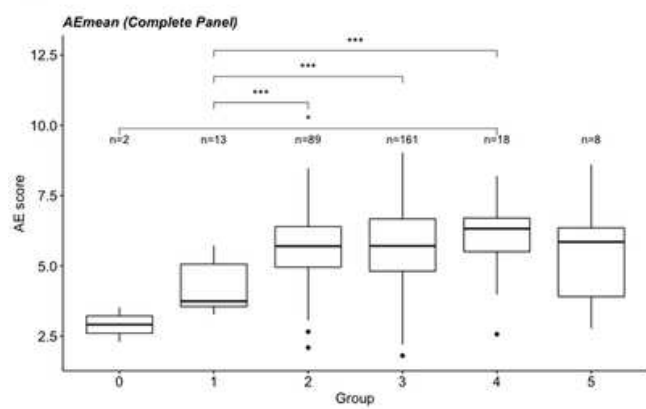

(d)

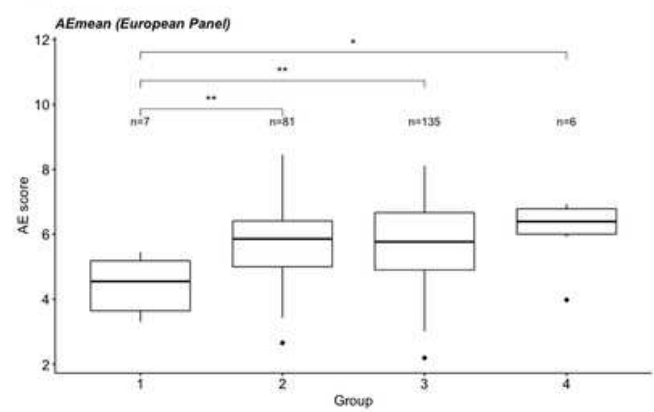

(b)

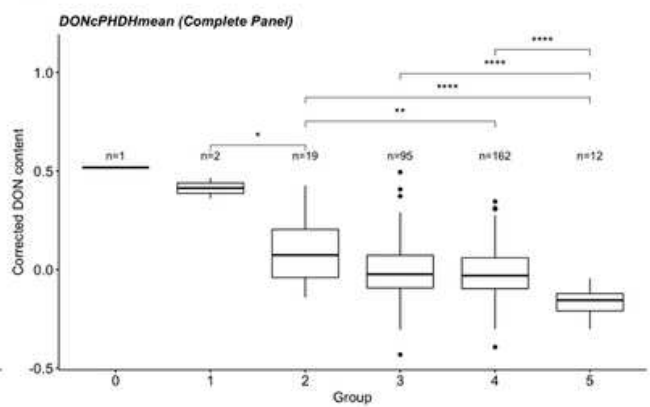

(e)

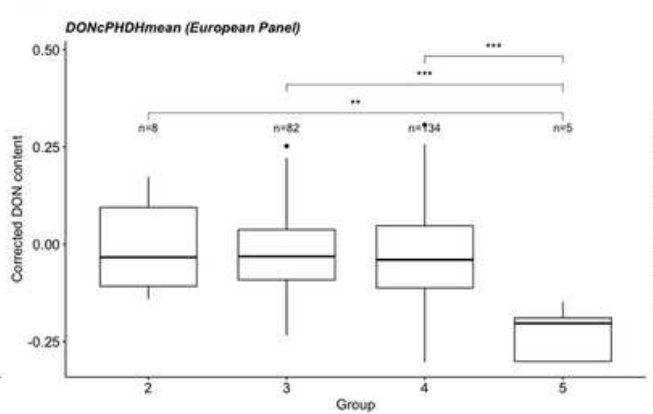

(c)

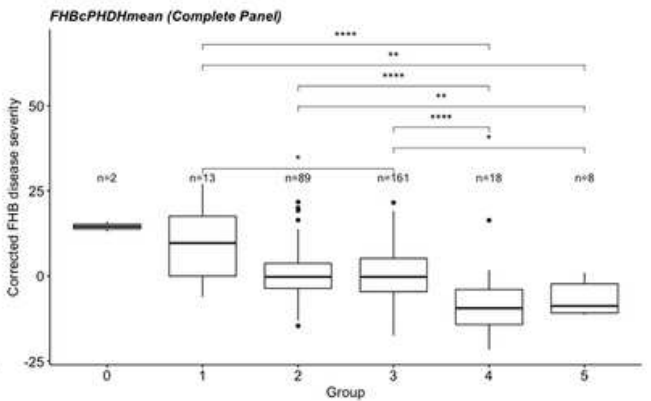

(I)

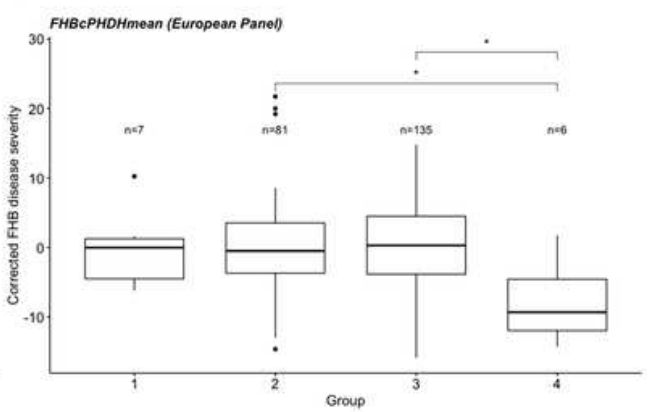

Figure 6

Boxplot showing the effect of number of stacked resistant alleles based on the a) mean AE, b) mean corrected DON content, c) mean corrected FHB disease severity of complete panel, and d) mean AE, e) mean corrected DON content, f) mean corrected FHB disease severity of European panel. Wilcoxon method is used for pair-wise comparisons ( ${ }^{\star \star *} \mathrm{P}<0.0001$, ${ }^{\star *} \mathrm{P}<0.001$, ${ }^{*} \mathrm{P}<0.05$, ns $>0.05$ ). Nonsignificant comparisons are not shown in this figure.

\section{Supplementary Files}

This is a list of supplementary files associated with this preprint. Click to download.

- OnlineResource1.xlsx

- OnlineResource2.docx

- OnlineResource3.xlsx 